\title{
Intra-pulpal temperature rises in power bleaching
}

\author{
Surface and intra-pulpal temperature rises during tooth bleaching: a study in vitro
}

M. Sulieman, M. Addy and J. S. Rees Br Dent J 2005; 199: 37-40

\section{Objective}

To measure the surface and intra-pulpal temperature increases in vitro on upper and lower anterior teeth during tooth whitening procedures. Method

A thermocouple was used to measure the temperature increase on the surface of an extracted upper central incisor tooth. Intra-pulpal temperature readings were made on upper and lower central incisors, lateral incisors and canines. Four lamps recommended for tooth bleaching were tested; a plasma arc lamp, a xenon-halogen lamp, a standard halogen lamp and a diode laser lamp. Temperature measurements were made with and without the bleaching agent present on the labial tooth surface.

Results

The increase in surface temperature readings ranged from $0.44^{\circ} \mathrm{C}$ (luma arc) to $86.3^{\circ} \mathrm{C}$ (laser) with no bleaching gel present. Intra-pulpal temperature increases ranged from $0.30^{\circ} \mathrm{C}$ to $15.96^{\circ} \mathrm{C}$. The presence of the bleaching gel reduced temperature increases seen at the tooth surface and within the pulp.

\section{Conclusions}

The increase in the intrapulpal temperature with most bleaching lamps was below the critical threshold of a $5.50^{\circ} \mathrm{C}$ increase thought to produce irreversible pulpal damage. The only lamp that produced an intrapulpal temperature increase above this threshold was the laser-based lamp and caution is advised when using this equipment.

\section{IN BRIEF}

- Bleaching gels act as effective insulators helping prevent large increases in pulpal temperatures.

- Most of the bleaching lamps tested did not raise the pulpal temperature to dangerous levels.

- Care needs to be taken when using laser based lamps at high power settings.

\section{COMMENT}

Tooth bleaching improves the whiteness and perceived aesthetic appearance of tooth tissue. This process often uses hydrogen peroxide (HP), either directly or via its generation in a carbamide peroxide gel. The use of peroxide based bleaching systems to whiten teeth remains controversial because of potential adverse effects on tooth structure and restorative biomaterials.

Additionally, in so called 'power bleaching' where a light source is used to accelerate the bleaching process, the resulting temperature rise at the tooth surface and hence at the pulp chamber may lead to pulpal necrosis.

This paper reported an investigation of surface and intra-pulpal temperature rises during power bleaching using four commercially available lamps (Apolite Plasma Arc, luma Arc, Optilux 501 and Diode laser). Temperature readings at the tooth surface of an extracted upper central incisor and within the pulp chamber of upper and lower central incisors, lateral incisors and canines with and without a 35\% HP gel on the labial aspect of the tooth were recorded using a thermocouple. The output of the thermocouple was fed into a data logger which was in turn connected to a laptop computer for data recording and analysis.

The results show increases in temperature at the tooth surface when the lamps are applied. The largest increase was $86^{\circ} \mathrm{C}$ with the diode laser lamp. The rise in temperature was markedly reduced when the bleaching gel was applied. There were smaller increases in intra-pulp temperature due to poor thermal conductivity of tooth tissue. Increases of $8.8^{\circ} \mathrm{C}$ and $10.7^{\circ} \mathrm{C}$ were recorded for the laser diode lamp with and without a gel respectively.

The main conclusion of the work was that a bleaching gel acts as an insulating layer against excessive temperature increases. The use of a laser diode on full power may cause pulpal necrosis; the threshold for necrosis was considered to correspond to an increase in pulp temperature of about $5.5^{\circ} \mathrm{C}$.

This paper has provided valuable preliminary data on the potential for thermal damage during 'power bleaching'. It certainly suggests that care should be taken when using this method, and the presence of a gel layer may be very important in terms of protecting the tooth and pulp. Further work is certainly required and, until this is reported, extreme caution is advised with respect to the use of a laser diode during tooth bleaching procedures.

S. K. Al-Salehi, Lecturer/Hon SpR in Restorative Dentistry, Department of Adult Dental Care, School of Clinical Dentistry, Sheffield doi: 10.1038/sj.bdj.4812555 